\title{
A low-complexity peak cancellation scheme and its FPGA implementation for peak-to-average power ratio reduction
}

\author{
Jiajia Song ${ }^{*}$ and Hideki Ochiai
}

\begin{abstract}
The power amplifier (PA) is the most power-hungry component in a wireless base station transmitter, and reducing the peak-to-average power ratio (PAPR) of wireless signals is an important issue for its effective use. In this paper, we focus on a field-programmable gate array (FPGA) implementation of the peak cancellation (PC) technique, which is known as the simplest method for PAPR reduction. The design issue of effective peak-cancelling pulses under the constraint on the out-of-band emission is addressed. In order to reduce its hardware complexity, a novel approach for generating peak-cancelling pulses is also presented. The experimental results based on long-term evolution (LTE)/LTE-Advanced and multi-band Wideband Code Division Multiple Access (WCDMA) signals demonstrate the validity of the proposed scheme. It has been shown that the proposed PC scheme can achieve lower in-band distortion than the conventional PC with an acceptable loss in out-of-band performance. Our study also includes mapping the signal processing methods onto a Xilinx virtex-7 FPGA device running at $245.76 \mathrm{MHz}$ and addresses the resource utilization and the hardware design in detail.
\end{abstract}

Keywords: PAPR reduction; Peak cancellation; FPGA; OFDM; LTE; LTE-Advanced; Multi-band; WCDMA

\section{Introduction}

Signals such as orthogonal frequency division multiplexing (OFDM) [1] and direct-sequence spread spectrum (DS-SS) for code division multiple access (CDMA) [2] are widely adopted in modern wireless communication systems due to their remarkable advantages such as flexible allocation of resources and high-spectrum utilization. As these signals are essentially a sum of multiple subcarriers/codes of multiple users in either frequency or time domain, the probability density functions (PDFs) of their signals tend to approach Gaussian, and thus, they exhibit high peak-to-average power ratio (PAPR) [3]. This poses strict demands on the dynamic range of data converters and especially limits efficient operation of the power amplifiers (PAs). Reducing the PAPR is hence important for boosting the PA efficiency by allowing higher average input power before saturation

*Correspondence: song-jiajia-kr@ynu.jp

Department of Electrical and Computer Engineering, Yokohama National University, 79-5 Tokiwadai, Hodogaya, Yokohama 240-8501, Japan occurs. To mitigate this issue, extensive studies have been performed [4-6].

Some of the PAPR-reduction techniques for Wideband Code Division Multiple Access (WCDMA) system can be found in, for example, $[7,8]$. On the other hand, those for multi-carrier and OFDM signals are much more abundant and appear in many forms including selected mapping (SLM) [9] and partial transmit sequence (PTS) $[10,11]$, just to mention a few. The aforementioned techniques do not incur distortion, but they either have a large computational complexity or have to modify the signal, which makes their implementation in high-speed real-time systems challenging or hinders standard-compliant operations. It should be noted that, as the digital techniques in a transmitter continue to scale, the power consumed by digital circuits also takes up a large portion of the total power consumption. Implementing the PAPR reduction with a high-speed and power-hungry digital signal processor (DSP) is obviously detrimental to the cost and power efficiency of the entire system.

\section{是 Springer}

(c) 2015 Song and Ochiai; licensee Springer. This is an Open Access article distributed under the terms of the Creative Commons Attribution License (http://creativecommons.org/licenses/by/4.0), which permits unrestricted use, distribution, and reproduction in any medium, provided the original work is properly credited. 
Motivated by the above observations, simple techniques such as clipping and filtering (CAF) [12-14], peak windowing (PW) $[7,15,16]$ and peak cancellation (PC) [17-19], which have much lower complexity, can be considered as more realistic approaches from the viewpoint of practical implementation. These techniques essentially introduce nonlinear operations so that distortions are inevitable. Given that some degree of distortion is generally allowed for the transmitted signals, such techniques are very attractive. The major drawback of CAF is the peak regrowth caused by the filtering effect, and the amount of regrowth is generally intractable. This is undesirable for a transmitter with digital predistorter (DPD), as the DPD needs to strictly keep the peak below a predefined value to ensure that no signal sweeps into the saturation region of the PA. Although PAPR regrowth can be somewhat alleviated by iterative use of CAF [20,21], the resulting complexity will be increased several fold because of the duplicated functional blocks. Furthermore, the latency issue becomes also prohibitive.

In contrast, $\mathrm{PC}$ is a much overlooked technique that has advantages in several aspects. PC simply generates independent cancelling pulses to cancel the peak values to a given threshold. It allows more cost-effective hardware implementation than the CAF as no filtering operation, which involves either a large number of multipliers or a bank of fast Fourier transform (FFT) blocks, is required. Moreover, PC can be easily configured to make compliant operation to signals of different communication standards. This is because the cancelling pulses can be updated to support a variety of carrier configurations and bandwidths. The concept of PC can be also used to facilitate generating cancelling pulses in $\mathrm{ACE}[22]$ and tone reservation (TR) [23,24]. In [24], the cancelling pulse is generated by performing inverse fast Fourier transform (IFFT) of the distorted signal after clipping and filtering located in the peak reduction tones (PTRs). This method thus generates neither in-band distortion nor out-of-band (OOB) emission but at a cost of data rate loss. In [19], the cancelling pulse generated using the PRTs is repetitively employed without FFT and IFFT for low-complexity implementation. Nevertheless, given the high computational complexity and high latency, the above-mentioned approaches may not be suitable for practical applications.

Until now, only a few papers address hardware implementation of the PC, and even fewer of them have mentioned its application to actual signals observed in commercial transmitters. Therefore, it is meaningful to investigate the applicability of $\mathrm{PC}$ in practical settings through field-programmable gate array (FPGA)-based experiments, and this is our main contribution in this work. Specifically, we investigate the feasibility and realizability of PC through elaboration of hardware design issues upon FPGA implementation. Furthermore, a novel
PC scheme with notably low-hardware complexity and improved error vector magnitude (EVM) performance is proposed with its effectiveness experimentally demonstrated.

This paper is organized as follows. Section 2 begins with the introduction of the basic model of PC considered throughout the paper, where the design of cancelling pulse is described. In Section 3, two conventional approaches with their respective advantages and drawbacks for implementing the PC are presented. Furthermore, a novel PC approach with much reduced hardware complexity is proposed and its implementation issues are discussed. Experiments using various signals are performed in Section 4 to demonstrate the benefits that can be achieved with the proposed PC scheme. Finally, our conclusion is given in Section 5.

\section{PAPR reduction by peak cancellation}

A basic diagram of the $\mathrm{PC}$ process considered in this work is sketched in Figure 1. Its principle is to generate cancelling pulses at the time instants where the peaks higher than the predetermined threshold are found. The generated pulses are linearly scaled and rotated with appropriate phase shift such that after their addition the original signals have the peaks reduced to the threshold [17].

\subsection{Peak-cancelling process}

To perform peak cancellation on the complex baseband signal, the target signal should be oversampled as the Nyquist-rate-sampled signals cannot correctly represent the actual amplitude of peaks of the continuous-time signals [3]. The discrete complex baseband signal $s_{n}$ has the general form of:

$$
s_{n}=r_{n} e^{j \theta_{n}}
$$

where $r_{n}$ and $\theta_{n}$ represent the amplitude and phase, respectively, at the $n$th time instant. Suppose that there are $N_{p}$ peaks that are larger than the predefined threshold $A_{t h}$ within a given time period $T$, and let $\rho_{1}, \rho_{2}, \cdots, \rho_{N_{p}}$ denote the corresponding successive peaks observed at the time instants $n_{1}, n_{2} \cdots, n_{N_{p}}$, respectively. Let $g_{n}$ denote the impulse response of the cancelling pulse centred at $n=0$, i.e. $g_{0}$ representing its maximum value. Then, the $i$ th peak cancelling pulse at the time instant $n_{i}$, where $i \in\left\{1, \cdots, N_{p}\right\}$, is expressed as:

$$
p_{n}^{(i)}=\left(r_{n_{i}}-A_{t h}\right) g_{n-n_{i}} e^{j \theta_{n_{i}}},
$$

where the phase is rotated by $e^{j \theta_{n_{i}}}$ to match the phase of the corresponding complex-valued peak sample, and the amplitude is scaled by $\left|r_{n_{i}}-A_{t h}\right|$ such that the peak value at $n=n_{i}$ is equal to $A_{t h}$ after peak cancellation. Then, the overall signal after cancellation of the entire peaks is expressed as: 


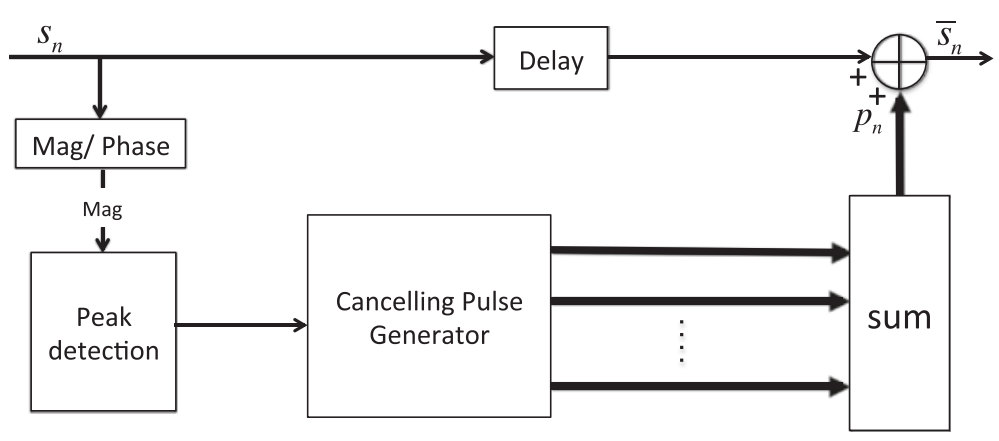

Figure 1 A simplified block diagram of the PC process.

$$
\begin{aligned}
\bar{s}_{n} & =s_{n}-\sum_{i=1}^{N_{p}} p_{n}^{(i)} \\
& =s_{n}-\underbrace{\left[\sum_{i=1}^{N_{p}}\left(r_{n_{i}}-A_{t h}\right) e^{\left.j \theta_{n_{i}} g_{n-n_{i}}\right]},\right.}_{=p_{n}},
\end{aligned}
$$

where $p_{n}$ is all the combined cancelling pulses located at the time instant $n_{i}$. If we ignore the change of the amplitude and average power due to the addition of all the cancelling pulses, $A_{t h}$ is the maximum amplitude after peak cancellation. In what follows, we refer to the corresponding PAPR determined by $A_{t h}$ as a target PAPR.

\subsection{Effect of the cancelling pulse}

The impulse response of the cancelling pulse $g_{n}$ determines the resulting OOB radiation. In general, $g_{n}$ should be compliant to the spectral mask of a given target standard. Suppose that $s_{n}$ is the oversampled version of the band-limited signal and let $J$ denote the oversampling factor such that the $\tilde{s}_{k J}$ represents the samples at the Nyquist rate for an integer $k$.

The resulting signal $s_{n}$ is then expressed as:

$$
s_{n}=\sum_{k} \tilde{s}_{k J} h_{n-k J}
$$

where $h_{n}$ is the corresponding impulse response of the pulse-shaping filter, and the summation is over the range of $k$ where the impulse response has a non-negligible effect. It is worth mentioning that even though the OFDM signal is not explicitly shaped by a filter, we can still find an equivalent form [25] to represent the virtual pulseshaping filter. Therefore, Equation 4 also applies to the conventional OFDM signals.

Now we consider the scenario where one peak $\rho_{i}$ is detected and subtracted by a cancelling pulse $p_{n}^{(i)}$. It then follows that:

$$
\begin{aligned}
\bar{s}_{n} & =s_{n}-p_{n}^{(i)} \\
& =\sum_{k} \tilde{s}_{k J} h_{n-k J}-\left[\left(r_{n_{i}}-A_{t h}\right) e^{j \theta_{n_{i}} g_{n-n_{i}}}\right] .
\end{aligned}
$$

Suppose that the peak position is precisely given at $n_{i}=k_{i} J+b_{i}$, where $k_{i}$ and $b_{i}$ are some integers. Then, Equation 5 is rewritten as:

$$
\begin{gathered}
\bar{s}_{n}=\sum_{k} \tilde{s}_{k J} h_{n-k J}-\left[\left(r_{n_{i}}-A_{t h}\right) e^{\left.j \theta_{n_{i}} g_{n-\left(k_{i} J+b_{i}\right)}\right]}\right. \\
=\sum_{k, k \neq k_{i}} \tilde{s}_{k J} h_{n-k J}+\left\{\tilde{s}_{k_{i} J}-\left(r_{n_{i}}-A_{t h}\right) e^{\left.j \theta_{n_{i}}\right\}} h_{n-k_{i} J}\right. \\
+\underbrace{\left(r_{n_{i}}-A_{t h}\right) e^{j \theta_{n_{i}}\left\{h_{n-k_{i} J}-g_{n-k_{i} J-b_{i}}\right\}}}_{d_{n}^{(i)}}
\end{gathered}
$$

which indicates that a proper design of $g_{n}$ will avoid the out-of-band emission, but we still observe that distortion component $d_{n}^{(i)}$ will affect all the other sampling instants. This distortion component can be nullified only if $g_{n}$ is set equal to $h_{n}$ and the peak position occurs at the Nyquist point, i.e. $h_{n-k_{i} J}=g_{n-k_{i} J-b_{i}}$.

In other words, if the cancelling pulse $g_{n}$ is identical to $h_{n}$, then no out-of-band regrowth will occur as the signal power is confined inside the pass-band of $h_{n}$. In fact, the clipping and filtering approach presented in [13] corresponds to this special case where $g_{n}$ is the periodic sinc function [25]. Since the periodic sinc function has nonnegligible impulse response over entire OFDM symbol, it causes considerable peak regrowth. Therefore, in practice, we wish to choose $g_{n}$ such that its side lobe (in time domain) vanishes rapidly, and yet, its frequency response has acceptable out-of-band emission in terms of adjacent channel leakage ratio (ACLR).

\subsection{Design of the cancelling pulse}

As we have seen, the impulse response of the peakcancelling pulse $g_{n}$, which is essentially a finite impulse response (FIR) filter, serves as a trade-off between the out-of-band radiation and in-band distortion. Specifically, 

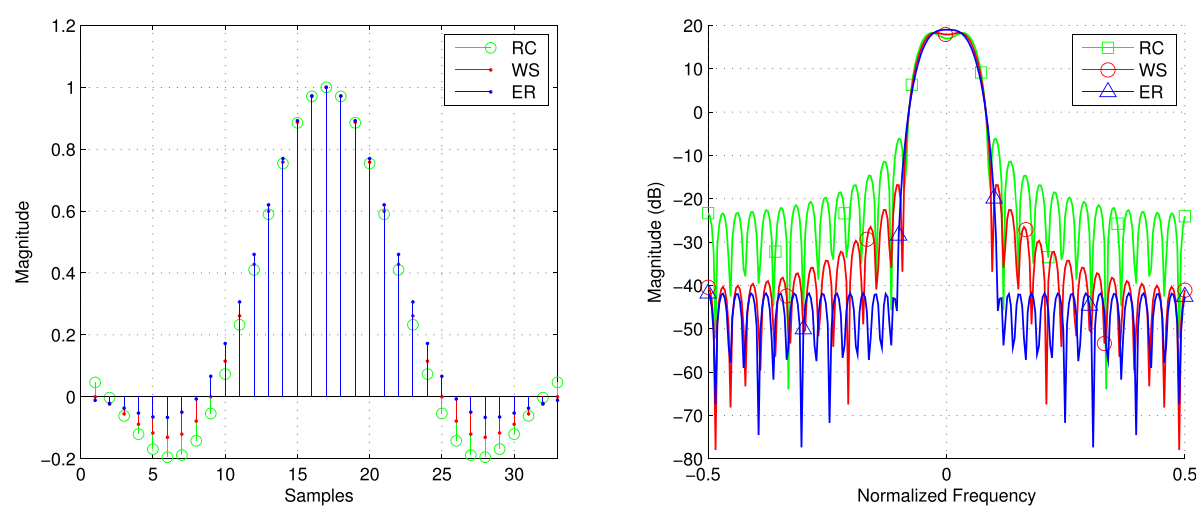

Figure 2 Performance comparison of the three different cancelling pulses designed based on different FIRs. The left hand side figure shows their impulse responses, whereas the right hand side figure shows the corresponding frequency responses. RC, raised cosine; WS, windowed sinc; ER, equal ripple.

shorter impulse response results in lower in-band distortion, but it will cause an increasing amount of outof-band radiation that may violate the specified spectral mask. Therefore, careful design of the cancelling pulse is essential.

However, there exists no solid algorithm or closedform deviation for finding the best cancelling pulse, and thus, exhaustive attempts are necessary to find the suitable one for a specified signal and to satisfy the design requirements. For instance, three different filters (cancelling pulses) of the same length are illustrated in Figure 2 with their respective impulse response and frequency response. Here, the windowed sinc (WS) is obtained by multiplying Kaiser window to sinc function. The raised cosine (RC) and sinc are both Nyquist filters as can be seen from the left hand of the figure. The equal ripple (ER) filter is obtained by the well-established Parks-McClellan algorithm [26] which minimizes the error in pass and stop bands by employing Chebyshev approximation. The performance of the peak cancellation based on the three cancelling pulses is demonstrated in Figure 3 using numerical simulation, where a WCDMA signal is used as its test signal. In this figure, as a practical measure for PAPR, we adopt the complementary cumulative distribution function (CCDF) of the instantaneous power normalized by its average power.

From the left hand side of Figure 3, we observe that similar PAPR performance is achieved. However, comparison of the power spectra in Figure 3 with their corresponding frequency responses in Figure 2 reveals that the frequency response of the cancelling pulses has the dominant effect on the resulting spectrum after peak cancellation.

The effects of pulse length on the in-band distortion (i.e. EVM) and out-of-band distortion (i.e. ACLR) are reported in Figure 4a,b, respectively. The measurement of EVM and ACLR follows the 3rd Generation Partnership Project (3GPP) frequency division duplexing (FDD) WCDMA downlink specification [27]. We have concluded
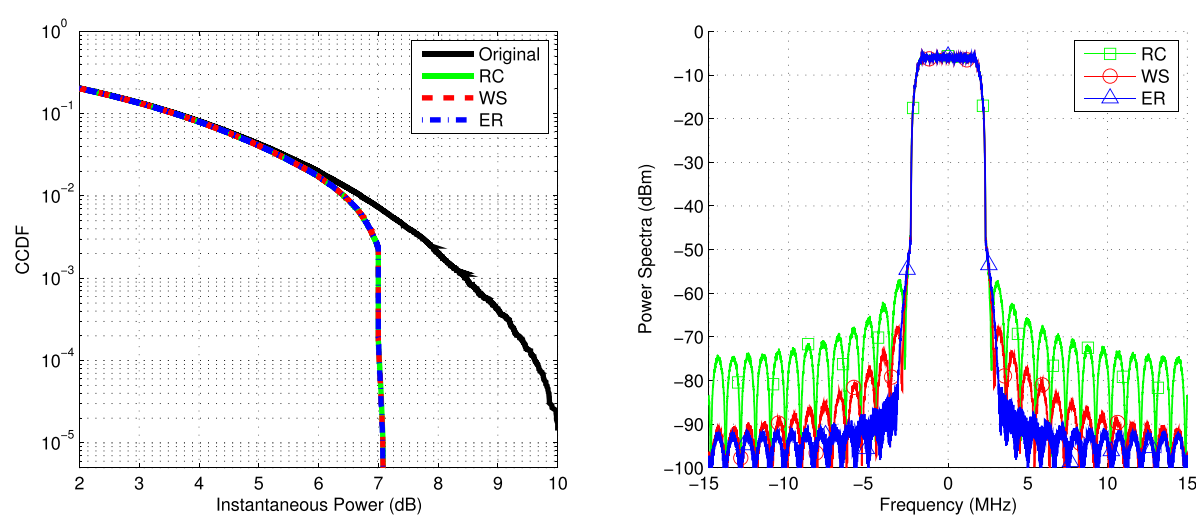

Figure 3 Peak-cancelled performance due to the different peak-cancelling pulses introduced in Figure 2. The left hand side figure shows the PAPR performance in terms of CCDF, whereas the right hand side figure shows the corresponding power spectra. CCDF, complementary cumulative distribution function; RC, raised cosine; WS, windowed sinc; ER, equal ripple. 


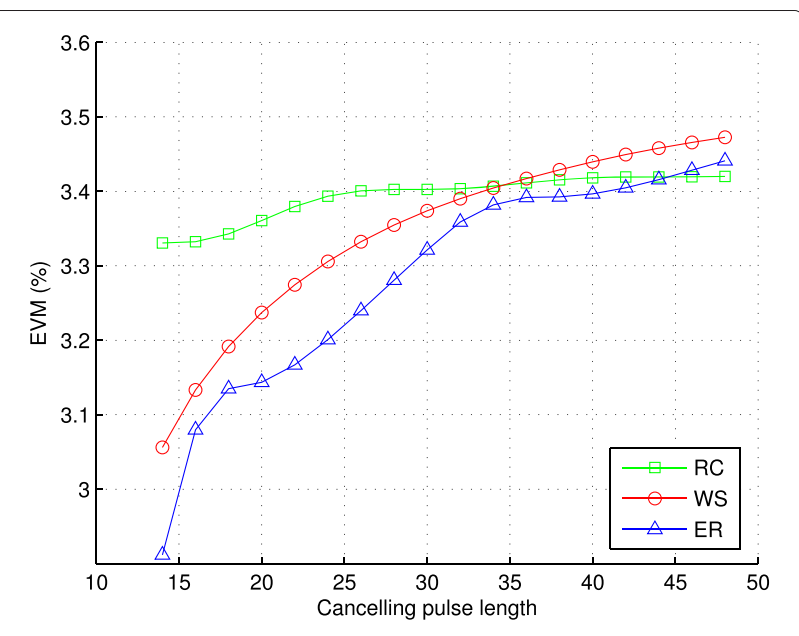

(a) Pulse length effect on EVM.

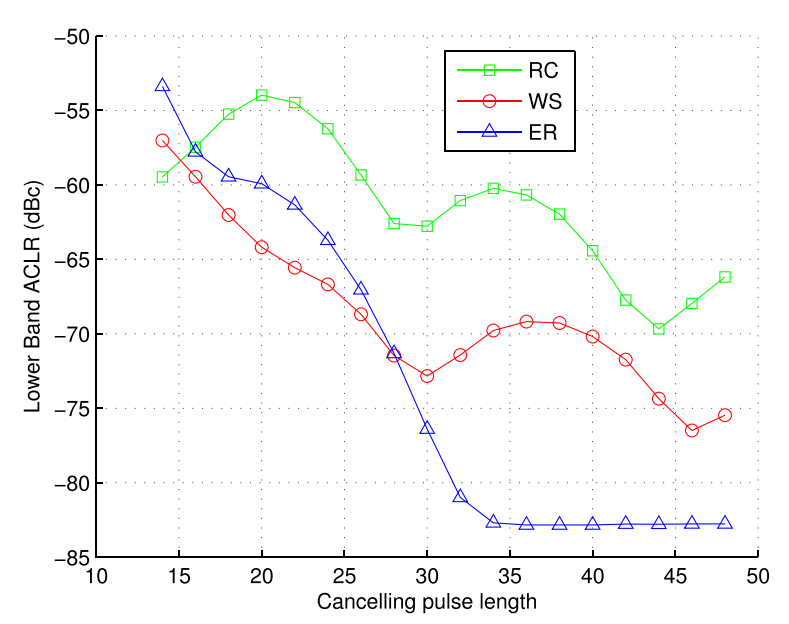

(b) Pulse length effect on ACLR.

Figure 4 Effect of different pulse length. (a) Pulse length effect on EVM. (b) Pulse length effect on ACLR. RC, raised cosine; WS, windowed sinc; ER, equal ripple; EVM, error vector magnitude; ACLR, adjacent channel leakage ratio.

in the last subsection that a longer pulse introduces more symbol errors to the target signal, and this is validated by Figure 4a. On the other hand, it can be easily grasped by inspection of Figure $4 \mathrm{~b}$ that ACLR reduces with longer cancelling pulse length. However, the curves shown in the figure have some fluctuations. This can be understood by inspecting Figure 5, where the impulse response for a typical low-pass filter is shown. Truncation length of the impulse response also affects the shape of the resulting frequency response. For instance, $L_{1}$ has worse out-of-band attenuation than $L_{3}$ but may be better than $L_{2}$, because $L_{2}$ has non-zeroes at the head and end. This heuristic observation reveals a basic clue for choosing the cancelling pulse length: the cancelling pulse should be as short as possible to minimize the distortion but should be long enough to give admissible ACLR.

It can also be observed from Figure 4a,b that the cancelling pulse generated by an equal ripple filter gives the best performance both in terms of EVM and ACLR. Better EVM performance is due to the lower side lobe in time domain, and lower ACLR is due to higher out-ofband attenuation of the cancelling pulse, as can be seen from Figure 2. It can also be seen from Figure 4a that even though the raised-cosine filter completely conforms to the pulse-shaping filter for WCDMA signal, it shows worst performance when the cancelling pulse length is short. This is mainly due to the distortion effect and the high sidelobe of the raised-cosine filter. In general, designing cancelling pulse with Parks-McClellan algorithm leads to better performance than other filter types that are frequently found in the literature.

\section{Hardware implementation of peak cancellation}

From this section and later, we focus on efficient implementation of PC by hardware. We first introduce the conventional approach for the implementation of $\mathrm{PC}$ as well as its alternative method, which is followed by the description of the proposed PC as well as their detailed implementation issues.

\subsection{Conventional implementation: scheme 1}

A conventional scheme for implementing the PC [28], which we refer to as a scheme 1 in what follows, is shown in Figure 6. The instantaneous magnitude and phase of the complex signal are computed with coordinate rotation digital computer (CORDIC) algorithm. The 'Peak Detect' block, which contains some registers and comparators, identifies the peak magnitude higher than the threshold. When a peak is detected, the corresponding phase and the magnitude amount higher than the threshold are latched. These values are used to scale and rotate the normalized real cancelling pulses, and these operations are accomplished by multiple CORDIC cores.

The cancelling-pulse generator block contains one counter, which is directly connected to the address port of the read only memory (ROM). The pre-determined cancelling pulse, which is compliant with the target signal spectrum, is stored in the ROM. The counter will be triggered as soon as one peak is found, and it is reset when it counts to the length of cancelling pulse $L$.

To deal with the occurrence of intensive peaks, multiple cancelling-pulse generators are necessary to generate independent cancelling pulses, and Figure 7 illustrates such an operation where the signal is propagating from left to right. When the first peak in the left is detected, the first generator will be turned on for $L$ clocks to generate the first cancelling pulse. In the event that the second and third peaks are detected when the first generator is 


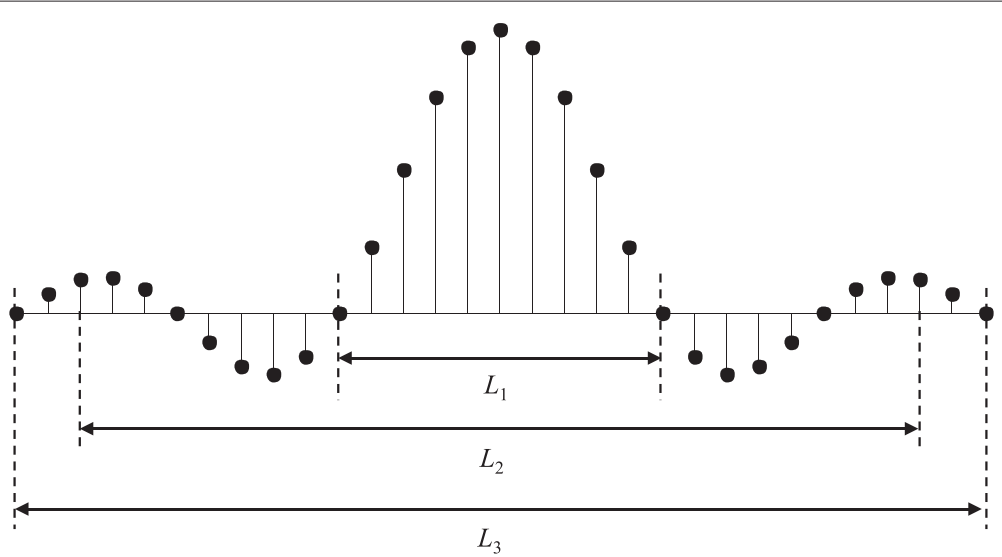

Figure 5 An example of typical impulse response of cancelling pulse with various truncation lengths.

still on, the second and third generators will be triggered to generate the second and third cancelling pulses. If the fourth peak is detected when ROM 1 is free, the first generator will be reused to generate the fourth cancelling pulse. In summary, when the previous cancelling-pulse generator is triggered and the next peak is found, the next cancelling-pulse generator will be triggered in sequel. This successive process continues until no peak higher than the threshold is found. All the outputs of the cancelling-pulse generators are summed and finally subtracted from the delayed original signal.

It becomes clear that the resource complexity of this scheme is bounded by the number of available cancellingpulse generators. Note that whether this scheme can cancel all the peaks in one pass or not depends on the specific parameters such as the required pulse length, targeted PAPR and the number of cancelling-pulse generators. It is easy to see that the generators in the upper side of Figure 6 have a higher probability to be used while the cancellingpulse generators in the bottom may be idle most of the time. Therefore, some compromise is necessary to determine how many cancelling-pulse generators are used. As a rule of thumb, five or six cancelling-pulse generators are enough when a reasonable threshold value is assumed, as the average number of the peaks above the threshold monotonically decreases as the threshold increases [3]. However, since the number of peaks itself is a random variable, with the fixed number of pulse generators, a failure to peak cancellation may occasionally happen. In this case, some iterative processing structure should be introduced, which may lead to increasing latency.

\subsection{Conventional implementation: scheme $\mathbf{2}$}

To overcome the issue of a peak cancellation failure, an alternative implementation scheme [29], which we refer to as scheme 2, can be applied. Let us rewrite Equation 3 as:

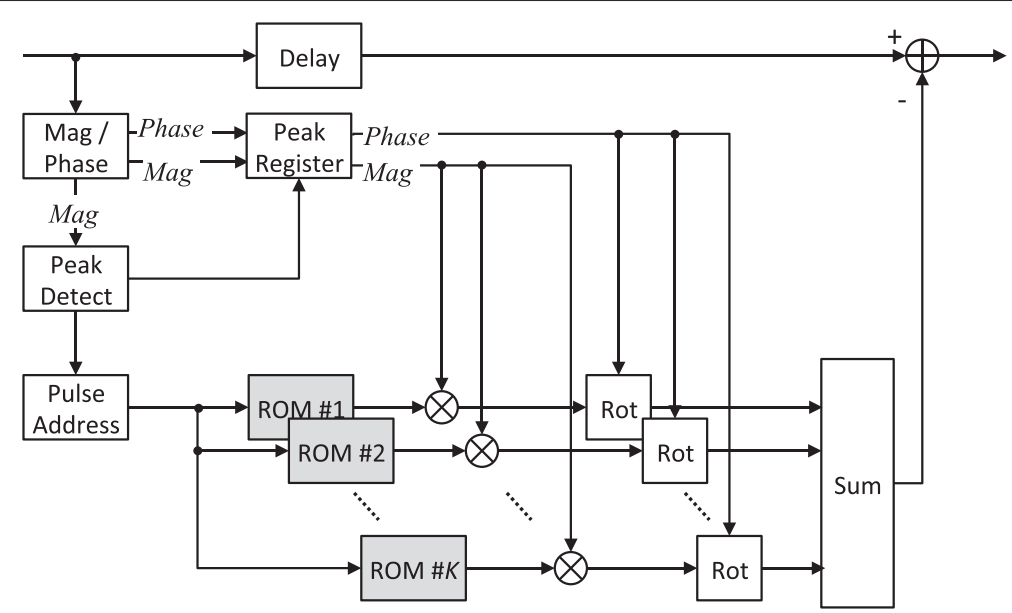

Figure 6 Architecture of conventional PC hardware implementation - scheme 1. ROM, read only memory. 


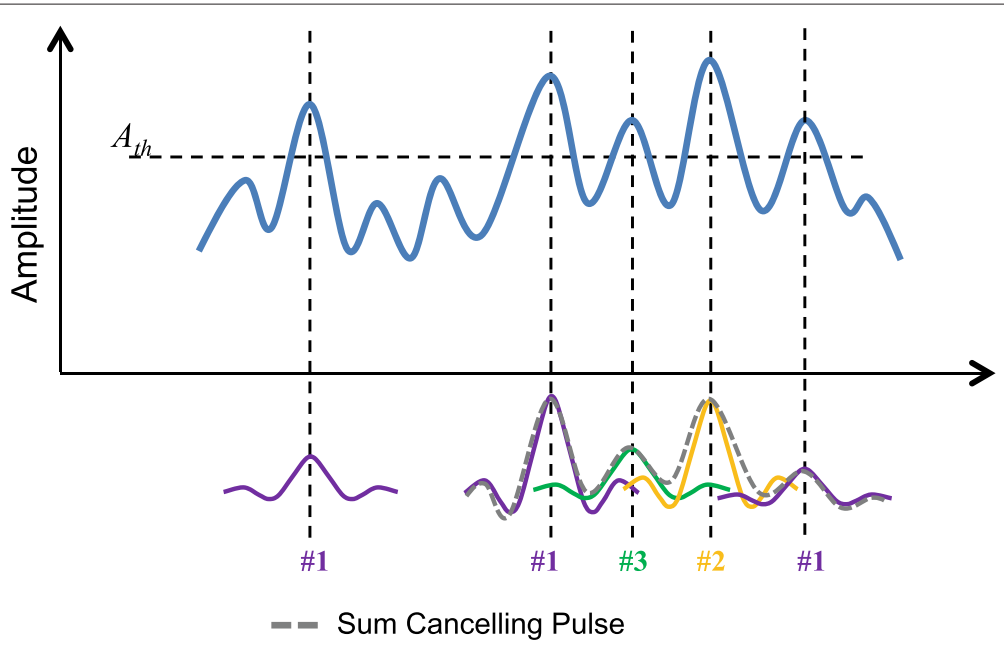

Figure 7 Principle of multiple cancelling pulse generation.

$$
\bar{s}_{n}=s_{n}-\sum_{i=1}^{N_{p}}\left[\left(r_{n_{i}}-A_{t h}\right) \cdot \delta\left(n-n_{i}\right) e^{j \theta_{n_{i}}}\right] * g_{n},
$$

where $g_{n}$ serves as the coefficients (impulse response) of an FIR filter and $*$ denotes the convolution operation. The cancelling pulses can be thus generated by propagating the delta function train to this filter, and this principle is illustrated in Figure 8 and its hardware implementation is given in Figure 9.

Similar to the first scheme, a CORDIC core is used to compute the magnitude and phase of the signal. The 'Pulse Gen' block outputs a sample which is properly scaled and rotated when a peak is detected. The resulting delta function train is complex-valued. It is easy to see from Figure 9 that the resource complexity relies primarily on the filter, while the complexity of its counterpart in Figure 6 depends on the number of CORDIC cores. Implementing an FIR filter requires a large number of multipliers, which makes the scheme 1 preferable as it consumes less resources.
In summary, the first scheme has lower complexity in terms of fewer multipliers but has the problem of peak generation failure. The second scheme is easier to implement but has higher resource complexity due to the use of FIR filter. In order to cope with the peak generation failure, however, the first scheme may require more iterations which in turn increase the complexity several fold. In this sense, the second scheme that has a fixed hardware overhead is preferred.

\subsection{The proposed peak cancellation}

As can be observed from Figure 7, a sum of multiple overlapped pulses forms the final pulse when intensive peaks occur. The tails of the previous pulses may happen to be added in-phase to the peaks in sequel, resulting in less effective peak reduction. Inspired by these observations, we proposed a novel approach of peak cancellation. The principle of the proposed approach is illustrated in Figure 10. Instead of generating complete cancelling pulses stored in the ROMs as the one shown in Figure 6, the proposed scheme generates truncated

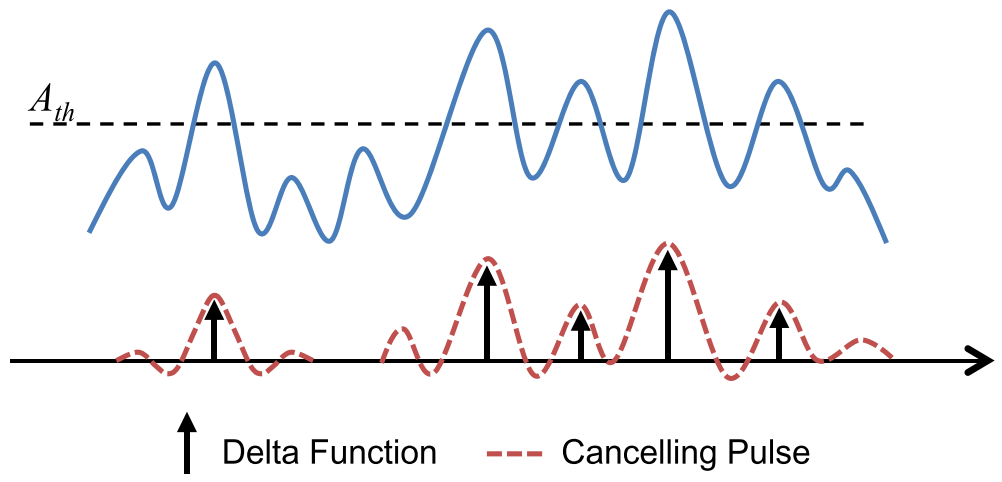

Figure 8 Principle of cancelling pulse generated by delta function trains. 


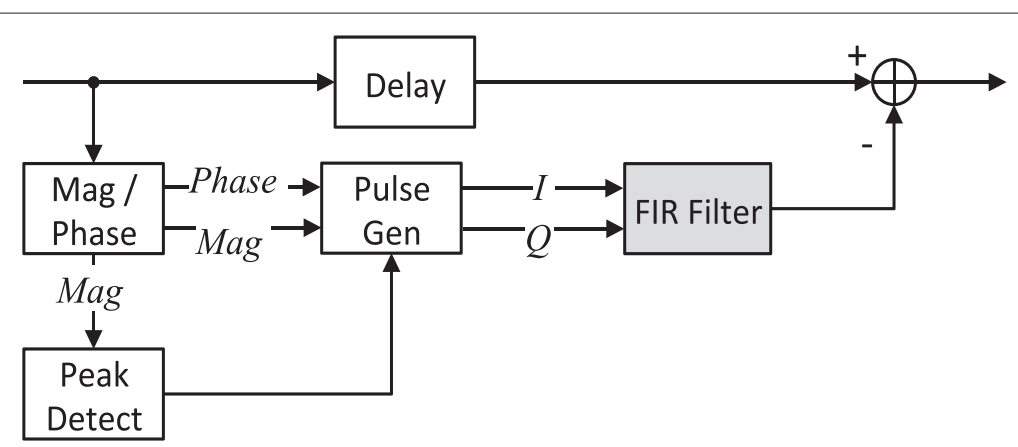

Figure 9 Architecture of conventional hardware implementation of peak cancellation - scheme 2 . FIR, finite impulse response.

cancelling pulses when they are overlapping with each other. More specifically, when the interval of two contiguous peaks is detected to be less than the predefined cancelling-pulse length, the generation of the first cancelling pulse terminates in the middle of the interval and immediately triggers the second cancelling pulse. Since this scheme reduces the length for overlapping cancelling pulses, lower in-band error can be expected in view of the observation given in the Section 2.3.

However, as can be seen from Figure 10, the cancelling pulses show obvious discontinuity which would produce substantial out-of-band emission. To mitigate the out-ofband emission, a filter can be applied to smooth the discontinuities, and the resulting waveform of the smoothed cancelling pulse is also denoted by the dashed curve in Figure 10. In our method, we use a simple moving average filter as a smoothing filter, with which the out-of-band spurious level caused by non-continuous cancelling pulses can be reduced. Note that the smoothing filter is actually optional as we can still obtain a moderate out-of-band emission without it, considering that the occurrence of high peaks is a rare event [3]. The smoothing filter is only necessary in the ACLR-prior circumstance. Normally,

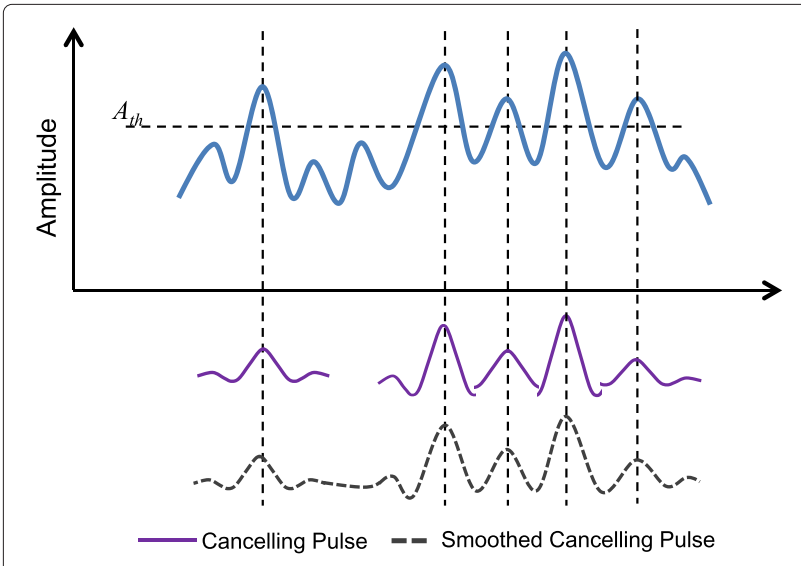

Figure 10 Principle of the proposed peak cancellation approach. a short-length smoothing filter is adequate to further improve the ACLR performance. As the filter length is very short, it gives negligible effect on EVM.

\subsection{Implementation of the proposed peak cancellation}

We now describe the hardware implementation aspects of the proposed scheme. A detailed block diagram of the proposed scheme is given in Figure 11. Some example waveforms of the internal signals labelled in Figure 11 are plotted in Figure 12.

Similar to the previous schemes, a CORDIC core is used to compute the instantaneous magnitude and phase of the signal. The 'Peak Detect' block, which contains some registers and comparators, outputs a ' 1 ' when a magnitude peak is found, as shown in the second plot in Figure 12. The output of the 'Peak Detect' block is connected to the enable ports of the two latches which store the magnitude and phase of the corresponding peak. The 'Interval Locator' block generates a ' 1 ' in the middle of two peaks when the interval of these peaks is less than the cancelling-pulse length. The outputs of 'Interval Locator' and 'Peak Detect' (I) and (II) are combined with an OR gate. A 'Delay' block is used to align these two signals as the 'Interval Locator' has a fixed delay. The 'Cancelling Pulse Duration" block produces enable signal (III) for the counter which outputs the address of the ROM. The counting direction of the counter is controlled by a latch output which is reversed when triggered by the output of the OR gate. Operation of these signals can be easily seen from the second and third plots in Figure 12.

The ROM output (V) is then scaled and rotated by the latched magnitude and phase to form the cancelling pulses and fed to the FIR filter. The smoothed cancelling pulses (VI) are subtracted from the delayed original signal to form the PAPR-reduced signal. It should be noted that the four 'Delay' blocks shown in Figure 12 do not necessarily indicate that the delay values for these blocks are identical.

The moving average filter used here for smoothing the truncated pulses is much shorter than the one used in 


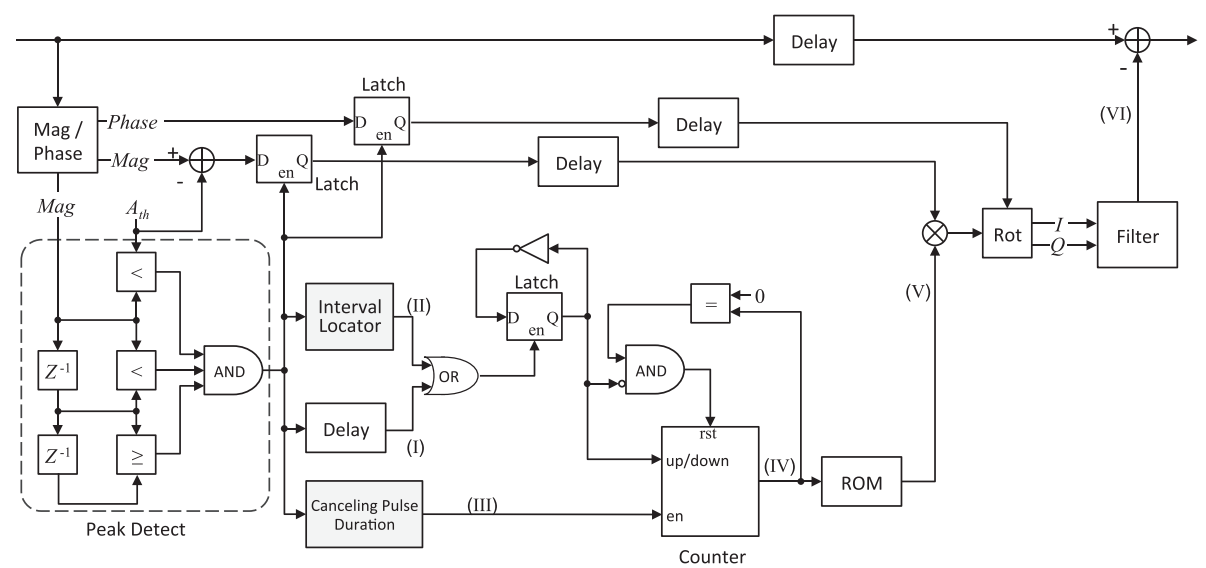

Figure 11 Hardware circuit of the proposed PC scheme using FPGA. ROM, read only memory.

Figure 9. Thus, it has less hardware complexity, even though both of them contain two CORDIC cores.

\section{Performance evaluation of the proposed peak cancellation}

In this section, the experimental results for implementation with FPGA are reported. We will compare the hardware complexity (in terms of resource utilization) of the proposed PC and conventional PC first. This is followed by the experimental results using a standard-conforming long-term evolution (LTE) signal as well as multi-standard signals.

\subsection{Experiment description}

The implementation is carried out using an FPGA evaluation board VC707, which contains a Xilinx Virtex-7
XC7VX485T-2FFG1761C device. The test WCDMA/LTE signal (baseband IQ) is generated by Matlab on the computer and is stored in a bank of RAMs in the FPGA as the signal source. The output of peak cancellation is captured by a series of integrated logic analyzers (ILAs) in parallel, which is arranged in a time-interleaved manner so as to receive the signal of long length. This signal is then transferred to computer through USB port and circularly shifted to align with the original signal and is analysed by Matlab.

A $245.76-\mathrm{MHz}$ clock is synthesized by the on-chip mixed-mode clock manager (MMCM), which uses the onboard $200-\mathrm{MHz}$ oscillator as the reference. The clock is set to integer times (64 times in our example implementation) of $3.84 \mathrm{MHz}$, targeting the specification of the 3GPP WCDMA and LTE signals.
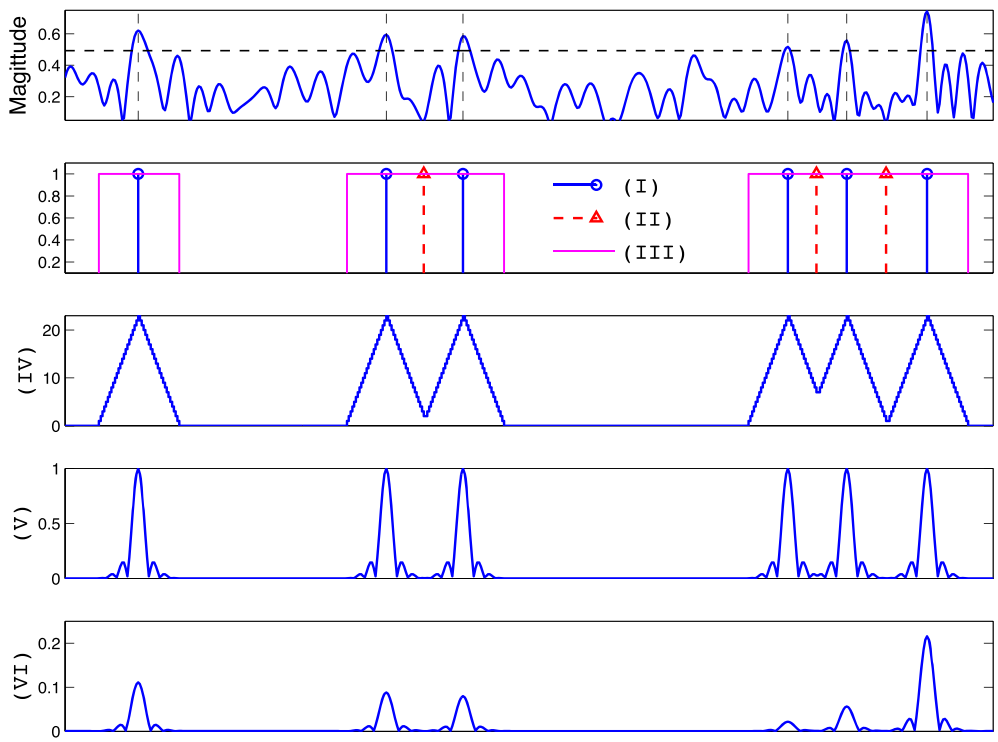

Figure 12 Internal signal illustration of the proposed PC circuit. 
The cancelling-pulse length is set to 115 , and the order of the moving averaging filter is set to 6 for the proposed PC. The complex cancelling pulse is assumed to support the asymmetric signal spectrum. As the smoothing filter is symmetric and one complex-variant multiplication uses three multipliers, the filter consumes $6 \div 2 \times 3$ multipliers. Furthermore, to optimize the speed, three multipliers are used to implement a CORDIC algorithm and the total of six multipliers by the two CORDIC cores. Another two multipliers are used to scale the cancelling pulse (real magnitude multiplying the complex cancelling pulse in $\mathrm{I} / \mathrm{Q}$ ) so that the total consumption of multipliers is 17.

\subsection{Resource utilization}

The FPGA resource utilization, which is evaluated with Resource Estimator using post-mapping, for the three PC approaches introduced are summarized in Table 1 where the scheme 1 is operated either without iteration or with one iteration. Only primary resources such as the slices and look-up tables (LUTs) are listed here, since they are generic for any FPGA, while the other resources used for our test (primarily block RAMs and IOs for ChipScope) are specific to the FPGA board and thus are not taken into consideration.

In scheme 1, the PC is comprised of four cancellingpulse generators, and therefore, four RAMs are used to store the cancelling pulse and accordingly four CORDIC cores are needed. One can see that with a single iteration, the hardware resource of scheme 1 is roughly doubled. It is obvious that all of the three schemes consume only a small portion of the resources such as flip flops (FFs) and slice LUTs. The conventional scheme 2 costs more multipliers. This is because it requires implementing the FIR filter to generate the cancelling pulses of equivalent length. For circuit integration, the multipliers are most concerned as they generally cost more power and take up larger area than other simple arithmetic elements. In this sense, the proposed PC is rather cost effective as it consumes fewer multipliers. Although the conventional scheme 1 without iteration requires even

Table 1 FPGA resource utilization table

\begin{tabular}{|c|c|c|c|c|}
\hline & FFs & Slice LUTs & $\begin{array}{l}\text { DSP48 } \\
\text { (Multipliers) }\end{array}$ & RAM \\
\hline Proposed & 3,920 & 3,439 & 17 & 1 \\
\hline $\begin{array}{l}\text { Conventional scheme } 1 \\
\text { (no iteration) }\end{array}$ & 3,354 & 3,117 & 15 & 4 \\
\hline $\begin{array}{l}\text { Conventional scheme1 } \\
\text { (one iteration) }\end{array}$ & 6,785 & 6,341 & 30 & 8 \\
\hline Conventional scheme2 & 5,749 & 5,383 & 171 & 0 \\
\hline Available in FPGA & 607,200 & 303,600 & 2,800 & 1,030 \\
\hline
\end{tabular}

FF, flip flop; LUT, look-up table. fewer multipliers, the peak-missing problem will make it unworkable as will be demonstrated in the subsequent subsections. In view of this, the proposed PC yields the lowest complexity.

The signal has 16-bit precision, which gives a noise floor of approximately $-80 \mathrm{~dB}$. Note that since the systems such as LTE and WCDMA require an ACLR of $-55 \mathrm{~dB}$ at most, actual implementation needs less bits.

\subsection{Test results using LTE signals}

The rest of the paper is devoted to the comparison of the three approaches in terms of in-band distortion, out-ofband emission and realizable PAPR.

The applied LTE signal is a 16-QAM OFDM signal with 1,200 subcarriers within $18.015-\mathrm{MHz}$ occupied bandwidth and $20-\mathrm{MHz}$ channel bandwidth. The basic sampling rate for such signal is $30.72 \mathrm{MHz}$ since the FFT size is 2,048. The PC for LTE signal is operated at 245.76 $\mathrm{MHz}$ which represents an oversampling rate of 8 . The signal spectrum and cancelling-pulse spectrum are demonstrated in Figure 13. The cancelling pulse used here was designed by Chebyshev approximation, as described in Section 2.3.

\subsubsection{Peak power reduction capability comparison for different $P C$ schemes}

In what follows, we evaluate the CCDF of the normalized instantaneous power after employing the PC schemes considered in this work.

The CCDF plots of the signal with the proposed PC and the two conventional $\mathrm{PC}$ schemes are demonstrated in Figure 14 where the target PAPR values are set as 6 and $8 \mathrm{~dB}$. As can be seen from the figure, the conventional scheme 1 without iteration exhibits high peaks due to

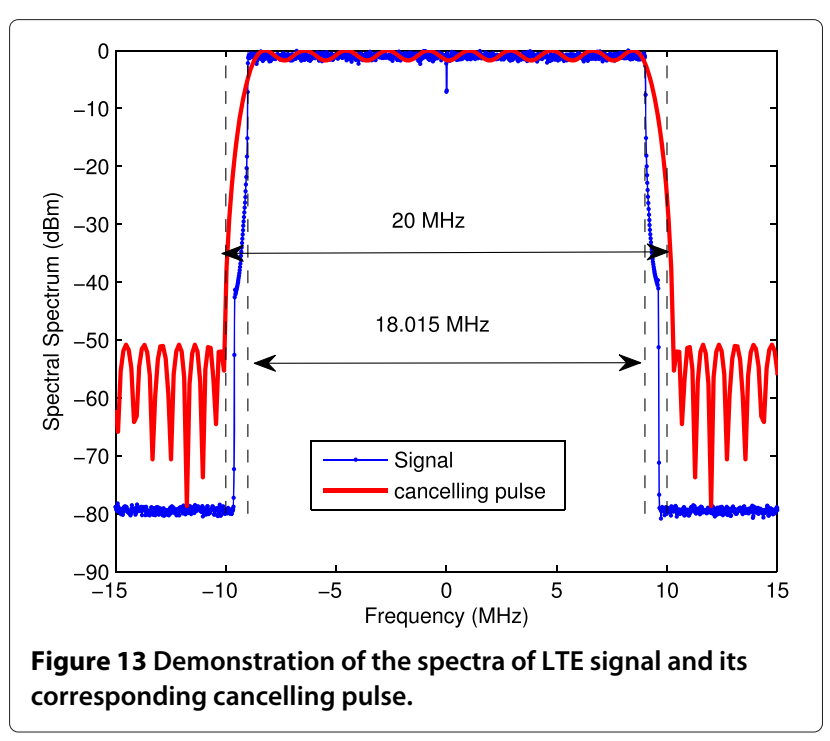




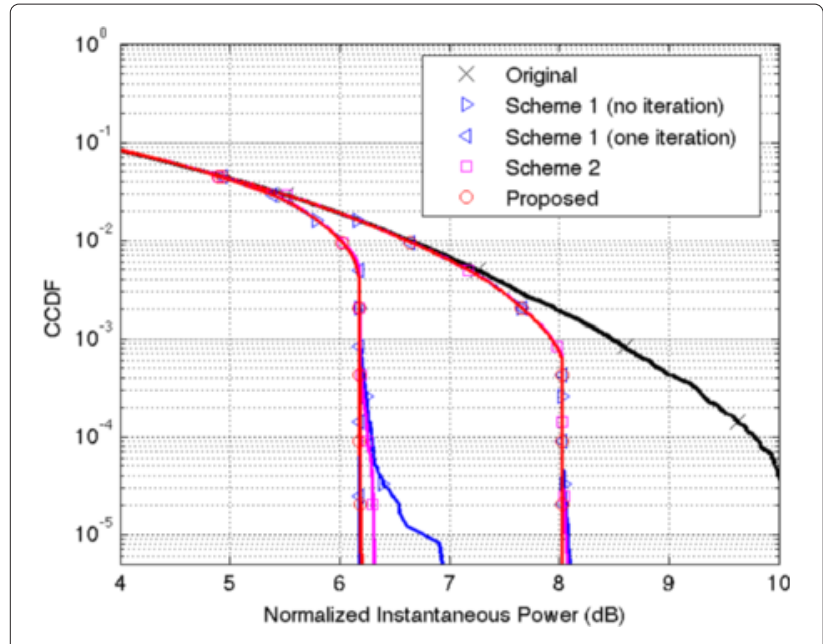

Figure 14 CCDF comparison in terms of normalized instantaneous power for different PC schemes with the target PAPR of $\mathbf{6}$ and $\mathbf{8} \mathbf{d B}$. CCDF, complementary cumulative distribution function.

the cancelling peak generation failure under the condition that only four ROMs are made available for this purpose. This problem can be solved by adding a single iteration, but this doubles the required resources as can be seen from Table 1 . The conventional scheme 2 outperforms the scheme 1 without iteration, at a cost of increasing hardware complexity. The proposed PC, on the other hand, achieves performance comparable to the scheme 1 with iteration, even with lower hardware complexity.

Considering the fact that the scheme 1 may not necessarily achieve a target peak power reduction performance without iteration, the experiments hereinafter will be focused exclusively on the proposed scheme and conventional scheme 2 .

An important factor for evaluating the performance of a PAPR-reduction technique is the actually realizable PAPR. It is well known that the CAF causes an unavoidable peak power regrowth, and this will make the precise control of PAPR challenging without resorting to increasing complexity such as iterative use of CAF [20], which may also introduce a prohibitive amount of latency. In contrast, the peak power achieved by PC schemes can reduce the effect of the peak power regrowth and thus achieve the PAPR close to the target PAPR. Figure 15 compares the peak power reduction capabilities of the schemes 2 and the proposed scheme. In this figure, in response to the target PAPR, the actual threshold values of the normalized instantaneous power at given specific CCDF of $10^{-4}$ and $10^{-5}$ are plotted as a realizable PAPR. It is observed that the proposed scheme outperforms the scheme 2 from the viewpoint of PAPR reduction as well.

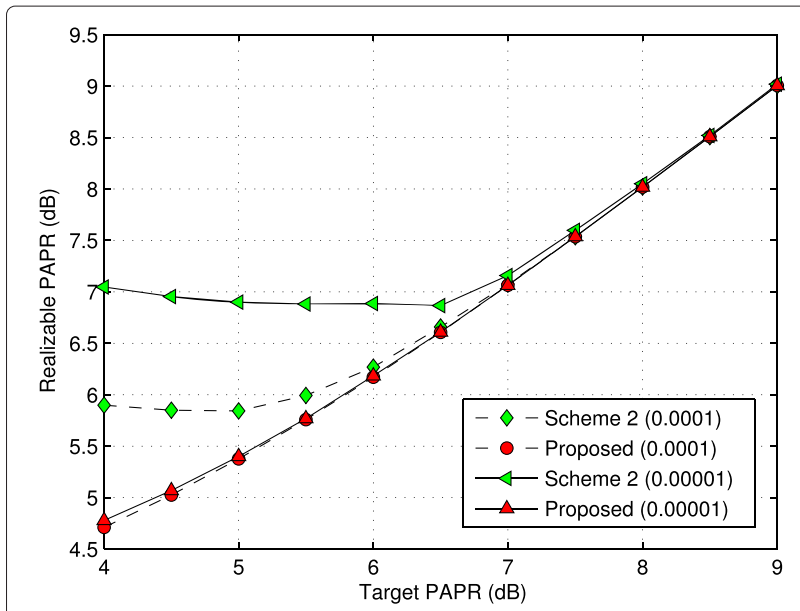

Figure 15 Realizable PAPR versus target PAPR for LTE signal. The realizable PAPR is defined as the corresponding threshold-normalized instantaneous power values measured at the CCDF of $10^{-4}$ and $10^{-5}$. PAPR, peak-to-average power ratio.

\subsubsection{In-band and out-of-band distortion comparison for different $P C$ schemes}

The constellation plots of the user data, which are 16QAM signal, with target PAPR of 5 and $7 \mathrm{~dB}$, are delineated in Figure 16. The pilot points (reference signal) in red, which is QPSK, are used to rotate, rescale and equalize the user data. It can be inspected from the figure that, for a target PAPR of $5 \mathrm{~dB}$, the constellation of the user data is rather dispersive.

The measured EVM and ACLR with different target PAPRs are demonstrated in Figures 17 and 18, respectively. It can be inspected from the figures that the proposed scheme yields lower in-band distortion (in terms of lower EVM) and higher out-of-band distortion (in terms of higher ACLR). This stems from the fact that since the proposed method generates shorter cancelling pulses, it can essentially reduce the in-band distortion in time domain but with the broader side lobes in frequency domain, as discussed in Section 2. Also, even though the proposed scheme has higher out-of-band emission, the observed ACLR with this parameter setting is still satisfactory for practical applications.

\subsection{Test results using various signals}

Finally, multiple tests have been performed to validate the proposed scheme for a more general framework with multi-carrier and multi-standard signals.

\subsubsection{Carrier-aggregated LTE signals}

The first test here assumes an LTE-Advanced signal with carrier aggregation of three $20-\mathrm{MHz}$ carrier components (CCs). The spacing of the two carriers are set to 20.1 $\mathrm{MHz}$, which is an integer multiple of $15 \mathrm{KHz}$ in order to maintain the OFDM subcarrier spacing. The target 


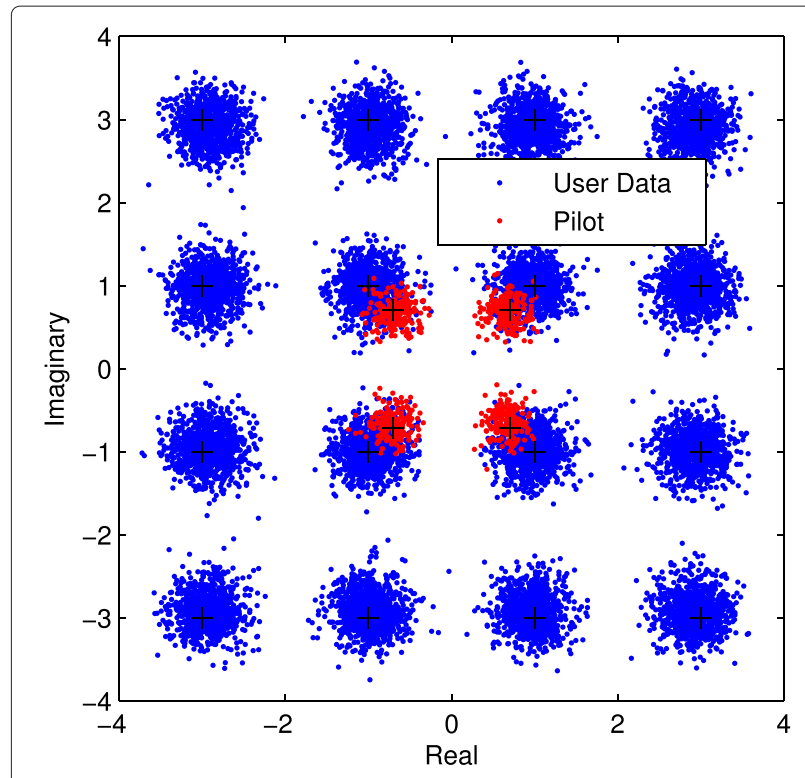

(a) when target PAPR $=5 \mathrm{~dB}$.

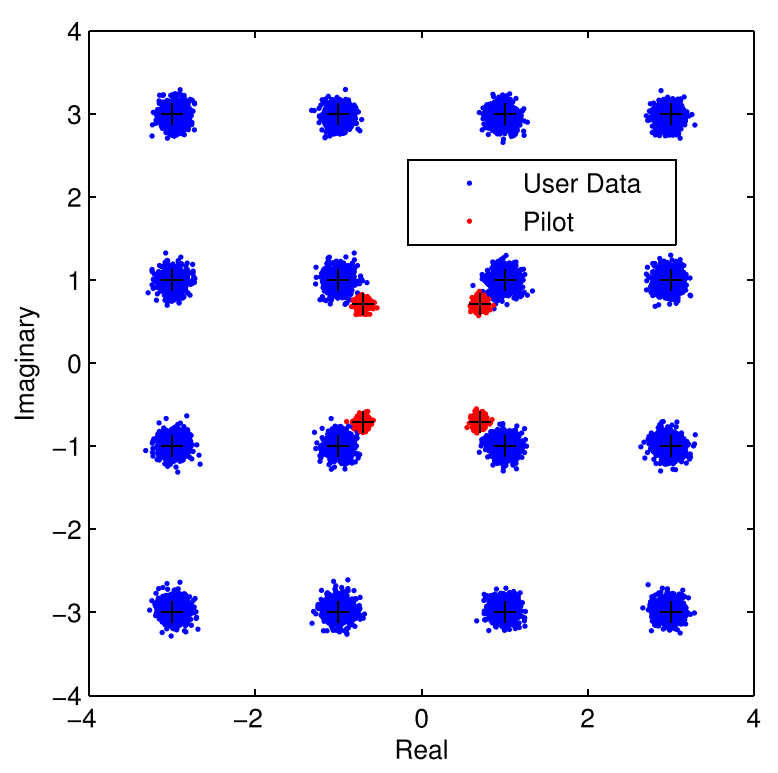

(b) when target $P A P R=7 \mathrm{~dB}$.

Figure 16 Constellation plots of user data. (a) When target PAPR = $5 \mathrm{~dB}$. (b) When target PAPR $=7 \mathrm{~dB}$. PAPR, peak-to-average power ratio.

PAPR is set to $7 \mathrm{~dB}$ which can yield reasonable EVM. The resulting CCDF is plotted in Figure 19a to show the effectiveness of the proposed scheme. The spectral density plots provided in Figure 19b show that, with the given cancelling pulse, the proposed scheme yields very limited spectral regrowth. The EVMs for the respective CCs under the condition of different realizable PAPRs are plotted in Figure 20. We observe that the EVM curves for

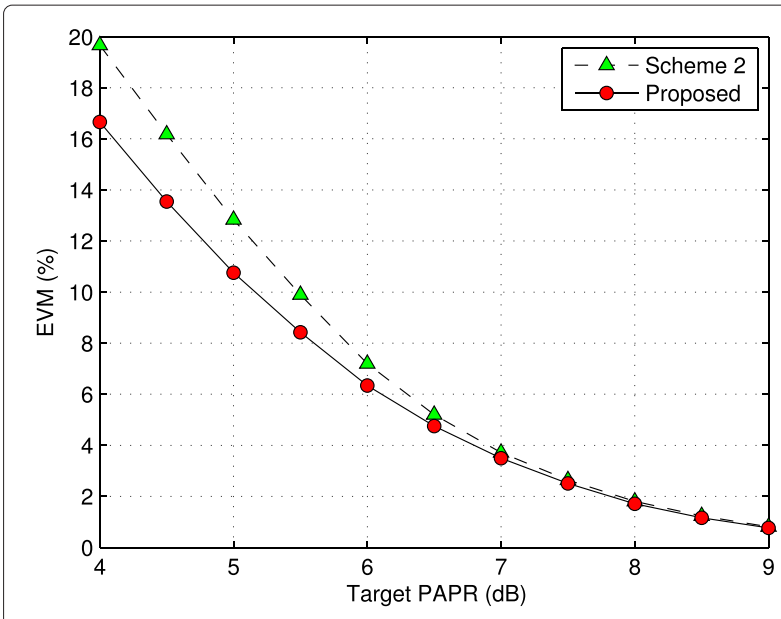

Figure 17 EVM versus target PAPR for LTE signal. EVM, error vector magnitude; PAPR, peak-to-average power ratio.

different CCs almost overlap with each other, indicating the effectiveness of the proposed PC under the system operated with multiple carrier frequencies.

\subsubsection{Multi-standard signals}

The second test uses a multi-standard signal, which contains three WCDMA carriers and a 20-MHz LTE carrier, where the two standard signals are spaced by $40 \mathrm{MHz}$. The WCDMA signal used in this paper is generated following the specification defined by 3 GPP test model 3 , for which 64 users (16-QAM) are multiplexed in dedicated physical data channel (DPDCH) to form the $3.84 \mathrm{Mc} / \mathrm{s}$ chip rate. The WCDMA chip is oversampled 64 times, and the PC is operated at a corresponding sampling frequency of 245.76 MHz.

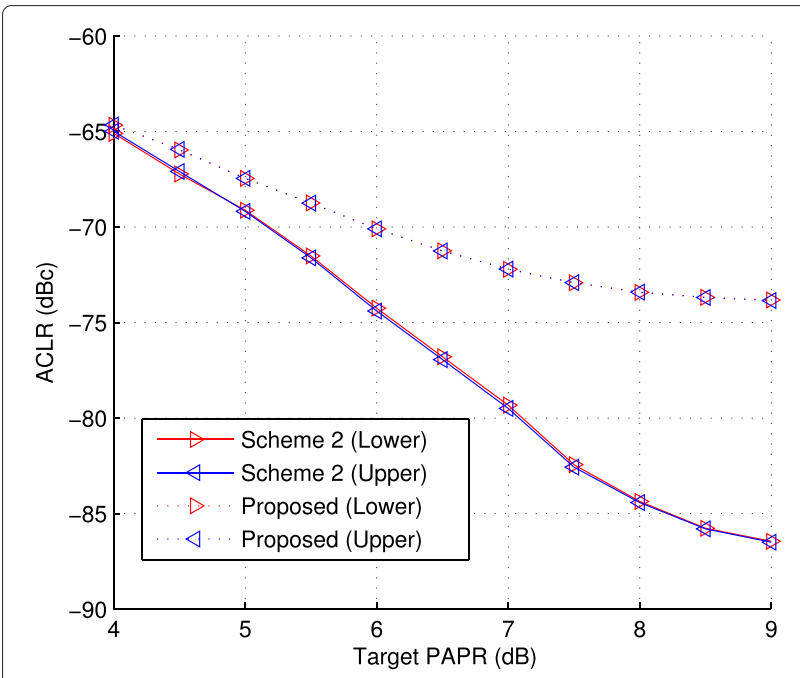

Figure 18 ACLRs (both lower and upper side bands) versus target PAPR for LTE signal. ACLR, adjacent channel leakage ratio; PAPR, peak-to-average power ratio. 


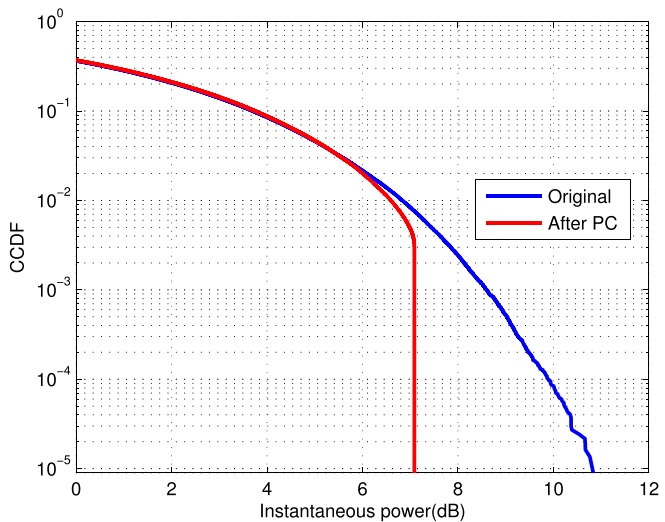

(a) CCDF when target PAPR is set to $7 \mathrm{~dB}$.

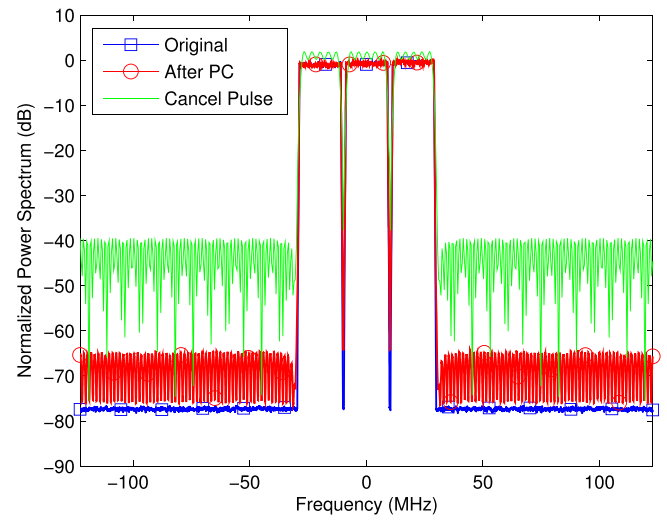

(b) The power spectral density plots when target PAPR is set to $7 \mathrm{~dB}$.

Figure 19 The performance comparison of $\mathrm{PC}$ for three-carrier LTE-Advanced signal. (a) CCDF when target PAPR is set to $7 \mathrm{~dB}$. (b) The power spectral density plots when target PAPR is set to $7 \mathrm{~dB}$. CCDF, complementary cumulative distribution function; $P C$, peak cancellation.

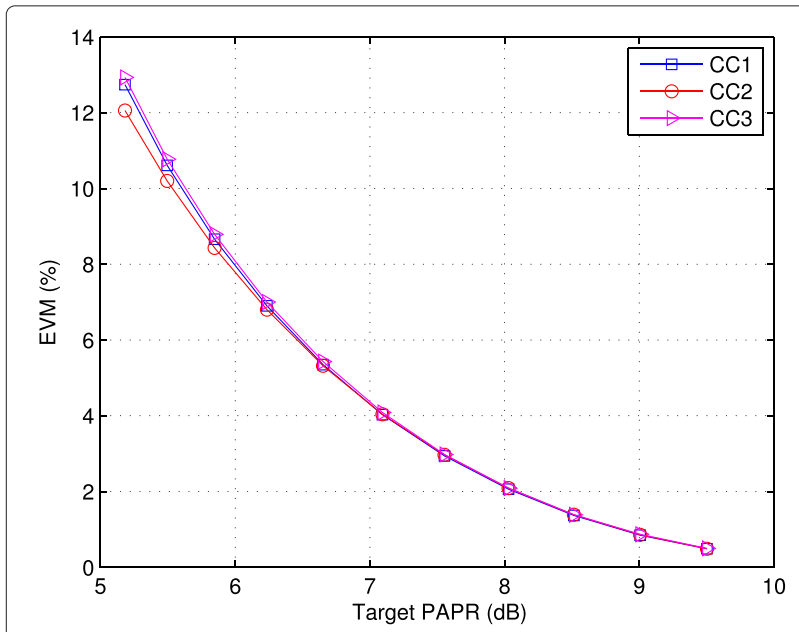

Figure 20 EVM of each LTE-Advanced component carrier. EVM, error vector magnitude; PAPR, peak-to-average power ratio.
The resulting CCDF and power spectral plots are given in Figure $21 \mathrm{a}, \mathrm{b}$, which demonstrate the adaptability of the proposed PC to the signal with non-contiguous spectrum. The proposed scheme, with a realizable PAPR of around $7 \mathrm{~dB}$, can meet the imposed requirement on power spectrum well as can be inspected from Figure 21b.

\section{Conclusions}

In this paper, the peak cancellation technique as a general purpose PAPR reduction has been addressed. The design issues of cancelling pulses that determine the performance of PC have been also discussed. Our main focus was on their FPGA implementation with a special consideration on the hardware complexity. A novel PC scheme with notably low-hardware complexity has been also presented. The experimental results using various signals have demonstrated the validity of the proposed approach.

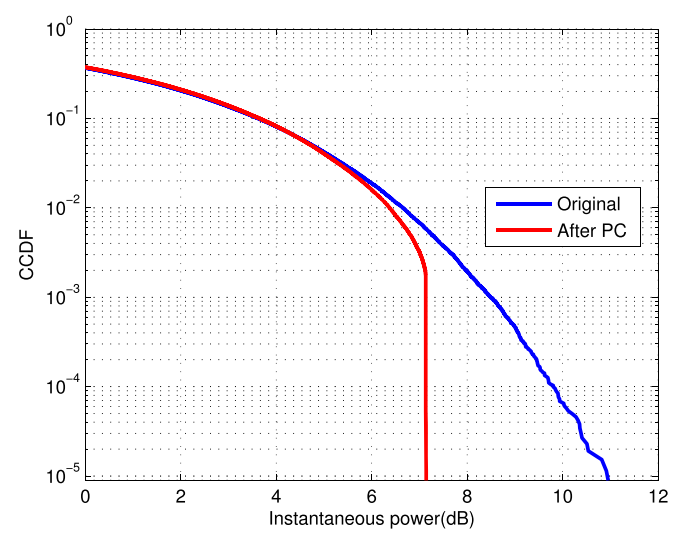

(a) CCDF performance when target PAPR is set to $7 \mathrm{~dB}$.

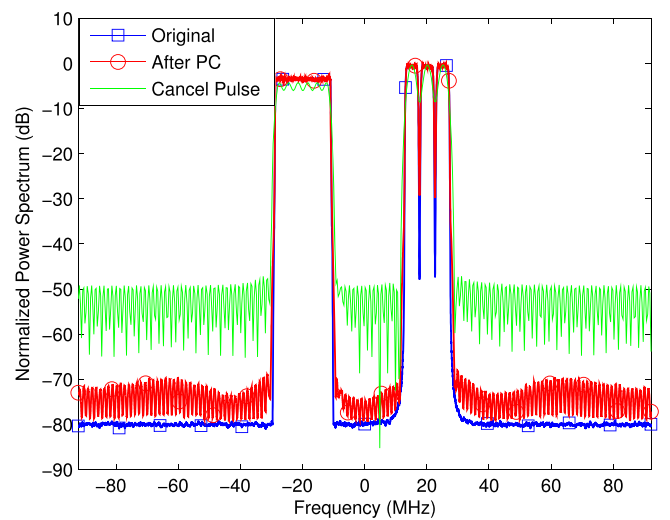

(b) The power spectral density plots when target PAPR is set to $7 \mathrm{~dB}$.

Figure 21 The performance comparison of PC for the aggregated signal of three-carrier WCDMA and LTE signal. (a) CCDF performance when target PAPR is set to $7 \mathrm{~dB}$. (b) The power spectral density plots when target PAPR is set to $7 \mathrm{~dB}$. CCDF, complementary cumulative distribution function; $\mathrm{PC}$, peak cancellation. 


\section{Competing interests}

The authors declare that they have no competing interests.

Received: 31 October 2014 Accepted: 4 March 2015

Published online: 24 March 2015

\section{References}

1. R van Nee, R Prasad, OFDM for Wireless Multimedia Communications. (Arthech House, Inc., Norwood, MA, USA, 2000)

2. H Honkasalo, K Pehkonen, MT Niemi, AT Leino, WCDMA and WLAN for $3 G$ and beyond. IEEE Wireless Commun. 9(2), 14-18 (2002)

3. H Ochiai, $\mathrm{H}$ Imai, On the distribution of the peak-to-average power ratio in OFDM signals. Commun. IEEE Trans. 49(2), 282-289 (2001)

4. SH Han, JH Lee, An overview of peak-to-average power ratio reduction techniques for multicarrier transmission. Wireless Commun IEEE. 12(2), 56-65 (2005)

5. T Jiang, Y Wu, An overview: peak-to-average power ratio reduction techniques for OFDM signals. Broadcasting IEEE Trans. 54(2), 257-268 (2008)

6. Y Rahmatallah, S Mohan, Peak-to-average power ratio reduction in OFDM systems: a survey and taxonomy. Commun. Surveys Tutorials IEEE. 15(4), 1567-1592 (2013)

7. O Väänänen, J Vankka, K Halonen, Simple algorithm for peak windowing and its application in GSM, EDGE and WCDMA systems. IEE Proc Commun. 152(3), 357-362 (2005)

8. W-J Kim, K-J Cho, SP Stapleton, J-H Kim, Doherty feed-forward amplifier performance using a novel crest factor reduction technique. Microwave Wireless Components Lett. IEEE. 17(1), 82-84 (2007)

9. RW Bauml, RFH Fischer, JB Huber, Reducing the peak-to-average power ratio of multicarrier modulation by selected mapping. Electron. Lett. 32(22), 2056-2057 (1996)

10. SH Müller, RW Bäuml, RFH Fischer, JB Huber, OFDM with reduced peak-to-average power ratio by multiple signal representation. Ann. Des Té, lécommunications. 52(1-2), 58-67 (1997)

11. RJ Baxley, GT Zhou, Comparing selected mapping and partial transmit sequence for PAR reduction. Broadcasting IEEE Trans. 53(4), 797-803 (2007)

12. $X \mathrm{Li}, \mathrm{LJ}$ Cimini, Effects of clipping and filtering on the performance of OFDM. Commun. Lett. IEEE. 2(5), 131-133 (1998)

13. H Ochiai, H Imai, Performance analysis of deliberately clipped OFDM signals. Commun. IEEE Trans. 50(1), 89-101 (2002)

14. R O'Neill, LB Lopes, in IEEE International Symposium on Personal, Indoor and Mobile Radio Communications (PIMRC'95). Wireless: Merging onto the Information Superhighway. Envelope variations and spectral splatter in clipped multicarrier signals. vol. 1 IEEE (Toronto, 1995) 71-75

15. HN Mistry, Implementation of a peak windowing algorithm for crest factor reduction in WCDMA Master's thesis, School of Engineering Science-Simon Fraser. University, Burnaby, BC, Canada, (2006)

16. M Pauli, H-P Kuchenbecker, Minimization of the intermodulation distortion of a nonlinearly amplified OFDM signal. Wireless Personal Commun. 4(1), 93-101 (1997)

17. T May, H Rohling, in IEEE Vehicular Technology Conference (VTC98). Reducing the peak-to-average power ratio in OFDM radio transmission systems, vol. 3 (IEEE Ottawa, 1998), pp. 2474-2478

18. L Dan, Y Xiao, W Ni, S Li, Improved peak cancellation for PAPR reduction in OFDM systems. Commun. IEICE Trans. E93-B(1), 198-202 (2011)

19. H-B Jeon, J-S No, D-J Shin, A new PAPR reduction scheme using efficient peak cancellation for OFDM systems. Broadcasting IEEE Trans. 58(4), 619-628 (2012)

20. J Armstrong, Peak-to-average power reduction for OFDM by repeated clipping and frequency domain filtering. Electron. Lett. 38(5), 246-247 (2002)

21. Y Wang, Z Luo, Optimized iterative clipping and filtering for PAPR reduction of OFDM signals. Commun. IEEE Trans. 59(1), 33-37 (2011)

22. BS Krongold, DL Jones, in Proceedings of 2003 IEEE International Conference on Acoustics, Speech, and Signal Processing (ICASSP'03). PAR reduction in OFDM via active constellation extension, vol. 4, (April 2003), pp. IV-525-8

23. J Tellado, JM Cioffi, PAR reduction in multicarrier transmission systems. ANSI Document, T1E1.4 Tech. Subcommittee. 4, 1-14 (1998)
24. L Wang, C Tellambura, Analysis of clipping noise and tone-reservation algorithms for peak reduction in OFDM systems. Vehicular Technol. IEEE Trans. 57(3), 1675-1694 (2008)

25. H Ochiai, On instantaneous power distributions of single-carrier FDMA signals. Wireless Commun. Lett. IEEE. 1(2), 73-76 (2012)

26. IW Selesnick, CS Burrus, Exchange algorithms that complement the Parks-Mcclellan algorithm for linear-phase FIR filter design. Circuits Syst. II: Analog Digital Signal Process. IEEE Trans. 44(2), 137-143 (1997)

27. ETSI 3rd Generation Partnership Project (3GPP). Base station conformance testing (FDD). TS 25.141, the European Telecommunications Standards Institute, September 2010

28. Inc Xilinx, LogiCORE IP Peak Cancellation Crest Factor Reduction v2.0. (Xilinx Inc., 1-28, 2009)

29. CA Azurdia-Meza, K Lee, K Lee, PAPR reduction by pulse shaping using Nyquist linear combination pulses. IEICE Electron. Express. 9(19), 1534-1541 (2012)

\section{Submit your manuscript to a SpringerOpen ${ }^{\circ}$ journal and benefit from:}

- Convenient online submission

- Rigorous peer review

- Immediate publication on acceptance

- Open access: articles freely available online

- High visibility within the field

- Retaining the copyright to your article

Submit your next manuscript at $>$ springeropen.com 